\title{
A compreensão oral e leitora dos níveis micro e macroestruturais de narrativas por indivíduos com lesão de hemisfério esquerdo e controles: um estudo piloto
}

The oral and reading comprehension of micro and macrostructural levels in narratives by left-brain-damaged patients and controls: a pilot study

Sabrine Amaral Martins ${ }^{1}$, Lisandra Rutkoski Rodrigues², Fernanda Loureiro

Doutoranda em Letras - Linguística, com ênfase em Neuropsicolinguística. É bolsista integral do volvimento) na Pontifícia Universidade Católica do Rio Grande do Sul (PUCRS). E-mail: sabrine.martins@acad.pucrs.br Doutoranda em Letras - Linguística, com ênfase em Neuropsicolinguistica. E bolsista integral do CNPQ (Conselho Nacional de Pesquisa e (Etólica do Rio Grande do SUl (PUCRS). E-mail: lisandra.rodrigues@acad.pucrs

Fonoaudióloga, doutora em Ciências da Saúde pela PUCRS. É bolsista de pós-doutorado da CAPES e professora colaboradora no Programa de Gerontologia Biomédica do Instituto de Geriatria
RESUMO: As lesões cerebrais advindas de acidentes vasculares encefálicos (AVEs) podem causar prejuízos na compreensão dos níveis de micro e macroestrutura de narrativas, acarretando dificuldades na vida dos indivíduos. Por isso, o presente artigo tem por objetivo apresentar dados de um estudo piloto que averiguou o desempenho de quatro indivíduos com lesão de hemisfério esquerdo e quatro indivíduos saudáveis na compreensão oral e leitora dos níveis micro e macroestruturais de narrativas. Foram aplicadas narrativas curtas, nas modalidades oral e escrita, seguidas de perguntas de compreensão. Na comparação entre os grupos e as modalidades, os resultados não mostraram diferenças estatísticas significativas. Qualitativamente, houve melhor desempenho de ambos os grupos nas tarefas de compreensão oral. Quanto à macroestrutura, o desempenho dos grupos foi inferior a 50\%. Os dados corroboram achados da literatura, que se mostram ainda inconclusivos quanto à compreensão leitora e oral de narrativas por indivíduos com lesão cerebral, e quanto ao impacto das duas modalidades de apresentação dos textos na compreensão.

Palavras-chave: Compreensão leitora; Compreensão oral; Lesão de hemisfério esquerdo; Narrativa; AVE.

ABSTRACT: Brain lesions due to cerebral vascular accidents (CVAs) can cause impairment to the comprehension of micro and macrostructural levels of narratives, leading to difficulties in people's daily lives. Therefore, the present article aims at presenting data from a pilot study that investigated the performance of four individuals with left-hemisphere damage and four healthy individuals in oral and reading comprehension of micro and macrostructural levels of narratives. Short narratives were presented in oral and written modes, followed by comprehension questions. In the comparison between groups and modes, the results did not show significant statistical differences. Qualitatively, both groups performed better in the oral comprehension tasks. As for the macrostructure, the groups' performance was inferior to $50 \%$. The data corroborate findings from the literature, which are still inconclusive regarding reading and oral comprehension of narratives by individuals with brain injury as well as the impact of the two modes in the presentation of the texts on comprehension.

Keywords: Reading comprehension; Oral comprehension; Left-brain damage; Narrative; CVA. 


\section{Introdução}

\ produção e a compreensão de narrativas permeiam a natureza humana.

Essa afinidade inicia já na infância, com a tradição oral de contação de histórias e segue até o cotidiano dos adultos, em que nos engajamos em atividades culturais que envolvem a produção e compreensão de narrativas diversas. Somos representados por narrativas individuais e, por vezes, autobiográficas, que constituem nossas memórias (GERGEN e GERGEN, 1988; HABERMAS e BLUCK, 2000). Sendo assim, a narrativa pode ser vista como um elemento de interação social diário e, logo, essencial à nossa vida em sociedade. Dessa forma, sendo constituinte da formação humana e de extrema importância, o processo de compreensão de narrativas deve ser investigado, principalmente quando se trata de indivíduos com lesão cerebral cuja habilidade de compreensão de narrativas pode estar comprometida, pois o acometimento na compreensão pode advir de um acidente vascular encefálico (SPRINGER, 2008).

Os danos na compreensão de narrativas podem se evidenciar nos níveis micro e macroestruturais ${ }^{1}$. As pesquisas sobre essa compreensão por indivíduos com LHE (lesão no hemisfério esquerdo) sugerem que a construção do nível macroestrutural pode estar preservada (GLOSSER e DESER, 1991; HUBER, 1990; ULATOWSKA e CHAPMAN, 1994). Sendo assim, atividades que envolvam os níveis micro e macroestruturais de narrativas podem ser trabalhadas nas sessões de reabilitação linguística, aprimorando o tratamento (ULATOWSKA e CHAPMAN, 1994). A compreensão desses níveis pode ainda ser influenciada pela modalidade em que os textos são apresentados. Todavia, os

\footnotetext{
${ }^{1}$ O nível microestrutural contém a informação local correspondente às palavras do texto e suas relações (van DIJK, 1976). Já o nível macroestrutural refere-se ao significado global do texto, implicado nas relações explícitas e implícitas entre suas proposições, determinando, assim, a organização temática e relaçôes explícitas e implícitas entr
}

estudos sobre o tema parecem inconclusivos sobre qual modalidade (oral ou escrita) pode influenciar a compreensão de narrativas. Sendo assim, o objetivo da pesquisa aqui apresentada foi investigar a compreensão oral e leitora dos níveis micro e macroestruturais de narrativas, por meio de um estudo piloto incluindo indivíduos com lesão encefálica no HE (hemisfério esquerdo) e controles saudáveis. Este estudo foi realizado de agosto a dezembro de 2016, na Pontifícia Universidade Católica do Rio Grande do Sul (PUCRS), como resultado da parceria entre o Grupo de Estudos em Neurolinguística e Psicolinguística (GENP) com o Hospital São Lucas, ambos da PUCRS.

\section{A compreensão oral e leitora por indivíduos saudáveis e com LHE}

Para a compreensão de um texto, é imprescindível relacionar o conhecimento linguístico e de mundo com as informações contidas nesse texto (KOCH e CUNHA-LIMA, 2004). Quanto mais relações forem estabelecidas, mais coerente será a representação desse texto e melhor será sua compreensão (van DEN BROEK, 1994). No gênero narrativo, foco deste trabalho, o processo de compreensão ocorre da mesma maneira. É preciso perceber as conexões entre as palavras, o conhecimento gramatical, e relacioná-los com a experiência prévia do indivíduo. As narrativas não se tratam somente de unidades de texto expandidas, como livros, podendo ser bastante curtas e ocorrer também na modalidade oral. Por isso, um prejuízo na compreensão, derivado de um comprometimento do $\mathrm{HE}$, pode levar a dificuldades sociais e profissionais para o indivíduo.

Na compreensão de narrativas por indivíduos saudáveis, há uma tendência de eles lembrarem mais acuradamente das ideias principais (WEGNER, BROOKSHIRE e NICHOLAS, 1984). Quando os ouvintes estão processando o discurso, eles procuram uma ideia principal com o objetivo 
de manter o texto coerente globalmente (van DIJK, 1976). Essa coerência global relaciona-se ao nível macroestrutural do texto, que não se atém ao significado de cada palavra em si, mas ao sentido global do texto. Durante o processo de compreensão, os detalhes costumam ser abstraídos para a formação de uma ideia principal (WEGNER, BROOKSHIRE e NICHOLAS, 1984).

A compreensão de narrativas por indivíduos com LHE parece se assemelhar à compreensão das populações de indivíduos saudáveis quanto ao processo de compreensão das ideias principais do texto e da coerência. Brookshire e Nicholas (1984) encontraram as mesmas características dos indivíduos saudáveis na compreensão oral de narrativas em indivíduos com LHE. Eles compreendiam e lembravam mais acuradamente das ideias principais do que dos detalhes das narrativas orais (BROOKSHIRE e NICHOLAS, 1984; NICHOLAS e BROOKSHIRE, 1993). Há dados apontando que a construção do nível macroestrutural do texto pode estar preservada no acometimento por uma lesão (DRESSLER e PLEH, 1988; GLOSSER e DESER, 1991; HUBER, 1990; ULATOWSKA et al., 1983; ULATOWSKA e CHAPMAN, 1991; ULATOWSKA, NORTH e MACALUSO-HAYNES, 1981). Outros reportam prejuízos do nível macroestrutural nos indivíduos afásicos (CHAPMAN e ULATOWSKA, 1992; PIERCE e GROGAN, 1992; ULATOWSKA et al., 1983; ULATOWSKA E SADOWSKA, 1992).

Ainda, devemos considerar as diferenças entre a compreensão desses níveis estruturais nas modalidades oral e escrita. Parece não haver um consenso sobre a eficiência de uma modalidade específica na compreensão (ROGOWSKY, CALHOUN e TALLAL, 2016). Alguns autores sugerem que a recordação após a leitura de um texto pode ser melhor do que a recordação após sua audição (DIXON et al., 1982; GREEN, 1981; LUND, 1991). Já outros sugerem não haver diferenças (MOYER, 2011; ROGOWSKY, CALHOUN e TALLAN, 2016).

Kintsch e Kozminsky (1977) afirmam que parece haver uma tendência de recordar um pouco mais a história quando o estímulo é oral do que quando é escrito (KINTSCH e KOZMINSKY, 1977). Embora seja um efeito muito sutil, os autores explicam que a modalidade oral pode levar a vantagens devido à natureza "oral" da memória de trabalho (KINTSCH e KOZMINSKY, 1977; SACHS, 1974).

Nos indivíduos com LHE, não foram encontradas evidências suficientes para sugerir que a compreensão de textos se beneficie de uma modalidade ou de outra. Algumas pesquisas sugerem que os indivíduos com LHE podem ser beneficiados na modalidade oral pela frequência superior com que ela é usada em relação à compreensão leitora (ORTIZ, 2010). Além disso, nas populações com baixa frequência de hábitos socioculturais como de leitura e escrita, a modalidade escrita apresenta-se como um obstáculo (ORTIZ; OSBORN e CHIARI, 1993). Por causa dessas impressões e da ausência de dados conclusivos, a pesquisa sobre a compreensão de texto nas modalidades oral e escrita em indivíduos com LHE se faz necessária. A seguir, o método da pesquisa é apresentado, incluindo-se a caracterização dos participantes.

\section{Método}

O estudo piloto consistiu em uma pesquisa de caráter experimental, transversal, com variáveis qualitativas e quantitativas. Para a avaliação dessas variáveis, foi feita uma comparação entre o desempenho em tarefas linguísticas, bem como o desempenho em testes neuropsicológicos de indivíduos com lesão focal no HE derivada de AVE há pelo menos quatro (04) meses $^{2}$, além de controles pareados por idade. Não foram incluídos participantes analfabetos.

2 A maior parte dos estudos avaliando desempenho linguístico com neuroimagem é desenvolvida a partir do quarto mês da ocorrencia do AVE Até este período, em partir do quarto mês da ocorrência do AVE. Até este periodo, em geral, as acomodações naturalmente associadas à recuperça difícil identificar se a melhora ocorreu devido a causas naturais, ou devido a algum tratamento para a
recuperação linguística. 
Esta pesquisa, aprovada pelo Comitê de Ética em Pesquisa da PUCRS, sob o número de registro no CAEE 51099415.6.0000.5336, faz parte do projeto guarda-chuva "Processamento discursivo, semântico e sintático na afasia: um estudo longitudinal com neuroimagem estrutural e funcional", coordenado pela professora Dra. Lilian Cristine Hübner, em parceria com colaboradores do serviço de neurologia do Hospital São Lucas da PUCRS.

\subsection{Caracterização dos participantes}

Participaram do estudo quatro (04) indivíduos com lesão única no HE, derivada de AVE isquêmico, sem depressão e declínio cognitivo e que foram previamente atendidos pela equipe de neurologia do Hospital São Lucas da PUCRS. Todos os participantes tiveram o episódio de AVE há pelo menos quatro (04) meses. Também participaram quatro (04) indivíduos saudáveis, configurando uma amostra por conveniência.

Os participantes foram convidados a responder o questionário sobre condições de saúde, aspectos sociodemográficos e socioculturais (adaptado de FONSECA et al., 2012), que continha o histórico das condições de saúde dos participantes, o Mini Exame do Estado Mental (MEEM - CHAVES e IZQUIERDO, 1992), o Inventário de dominância manual de Edinburgh (BRITTO et al., 1989), o questionário de status socioeconômico (ABEP Associação Brasileira de Empresas e Pesquisa, 2015), escala de depressão geriátrica (GDS - ALMEIDA e ALMEIDA, 1999), questionário sobre aspectos socioculturais pré e pós-lesão (PAWLOWSKI, 2012). Abaixo, as Tabelas 1 e $\mathbf{2}$ apresentam as características dos participantes.

Tabela 1 - Características dos participantes com LHE

\begin{tabular}{|c|c|c|c|c|c|c|c|c|}
\hline Participantes & Idade & Sexo & Data AVE & Instrução & Profissão & Local lesão & MEEM & SSE \\
\hline P01 & 62 & $\mathrm{~F}$ & $29 / 09 / 2015$ & Fundamental completo & Costureira & ACME e TE & $23 / 30$ & B2 \\
\hline P02 & 75 & $\mathrm{~F}$ & $05 / 02 / 2016$ & $2^{\text {a }}$ série Fundamental & Costureira & $\mathrm{TE}$ & $26 / 30$ & B2 \\
\hline P03 & 66 & M & $19 / 08 / 2015$ & $4^{\mathrm{a}}$ série Fundamental & Mecânico & Caudato e TE & $27 / 30$ & $\mathrm{C}$ \\
\hline $\mathrm{P} 04$ & 60 & $\mathrm{~F}$ & $16 / 03 / 2016$ & 6a série Fundamental & Vendedora & CIE & $27 / 30$ & $\mathrm{C}$ \\
\hline
\end{tabular}

P: Participantes; AVE: Acidente Vascular Encefálico; F: Feminino; M: Masculino; MEEM: Mini Exame do Estado Mental; SSE: status socioeconômico; ACME: Artéria Cerebral Média Esquerda; TE: Tálamo Esquerdo; CIE: Cápsula Interna Esquerda. Fonte: Autoras.

Tabela 2 - Características dos participantes controles

\begin{tabular}{ccccccc}
\hline Controles & Idade & Gênero & Instrução & Profissão & MEEM & SSE \\
C01 & 61 & F & Médio completo & Costureira & $29 / 30$ & B2 \\
C02 & 66 & M & Média completo & Motorista & $28 / 30$ & B2 \\
C03 & 64 & F & 4⿳亠丷a série Fundamental & Do lar & Não aplicado & B1 \\
C04 & 60 & F & 2a série Fundamental & Serviços Gerais & $25 / 30$ & C \\
\hline
\end{tabular}

C: Controles; F: Feminino; M: Masculino; MEEM: Mini Exame do Estado Mental; SSE: Status socioeconômico. Fonte: Autoras. 
A pesquisa contou com participantes destros, com hábitos de leitura de revistas ou jornais pouco frequentes. Segundo eles, ler informações nas redes sociais uma vez por dia é seu hábito de leitura mais frequente. É importante ressaltar que as profissões que tanto o grupo GLHE (grupo com lesão no hemisfério esquerdo) quanto o grupo GC (grupo controle) possuíam não requeriam hábitos de leitura frequentes.

Para a caracterização dos participantes, também foi utilizado um critério de classificação econômica para famílias brasileiras da Associação Brasileira de Empresas de Pesquisa - status socioeconômico (SS). Esse questionário estima a classe social e não a renda das famílias. O levantamento é comumente tratado como Critério Brasil - CCEB - e é constituído de perguntas sobre o que há dentro de casa e a quantidade de itens de determinadas categorias. No total, são 10 itens como: televisão, rádio, banheiro, automóvel, empregada mensalista, máquina de lavar e secar, computador, DVD, geladeira e freezer, além da descrição da pavimentação da rua onde é a residência do entrevistado e o tipo de fornecimento de água. A partir desses dados, são obtidas classificações em: A1, A2, B1, B2, C1, C2, D e E.

Os participantes deste estudo piloto foram classificados nas categorias B1, B2 e C1. A classificação B, no geral, indica um maior potencial de consumo para as famílias, juntamente com a primeira categoria. Ambas correspondem a uma parcela de $14 \%$ da população brasileira, conforme Kamakura e Mazzon (2013). O potencial de compra de bens e serviços dessas categorias fica em torno de $58 \%$. As famílias inseridas na categoria C apresentam um potencial de consumo menor, em torno de $26 \%$. Cerca de $16 \%$ da população brasileira insere-se nessa categoria. Esse status socioeconômico da amostra pode indicar um acesso mais recorrente a hábitos socioculturais como escolas, cursos extracurriculares, viagens, livros, dentre outros, influenciando diretamente nas habilidades averiguadas neste estudo (KAMAKURA e MAZZON, 2013).
Na próxima seção, detalharemos a confecção das tarefas e os procedimentos para coleta e análise dos dados.

\subsection{Tarefas}

Foram desenvolvidos oito (08) textos com temáticas comuns ao dia a dia. Os textos continham aproximadamente 100 palavras e oito (08) sentenças (ver ANEXo I-A) e índices Flesch de leiturabilidade semelhantes aos textos da bateria Montreal Toulouse Brasil (MTL-BR) (PARENTE et al., 2016), conforme as medidas dadas pelo software CoH-Metrix-Port (ALUISIO et al., 2010), único critério de leiturabilidade disponível para o português brasileiro $^{3}$. Também foram usados os dois (02) textos presentes na bateria Montreal Toulouse Brasil (MTL-BR) (PARENTE et al., 2016). 0 primeiro texto da bateria tratava-se do texto para a tarefa de compreensão oral e o segundo tratava-se do texto apresentado pela bateria para a tarefa de compreensão leitora.

Os textos desenvolvidos para esta pesquisa e as seis (06) perguntas de interpretação para cada um foram submetidos ao julgamento de nove (09) juízes psicolinguistas com experiência em estudos experimentais. A partir das avaliações dos juízes, dois (02) textos foram escolhidos para treino em cada modalidade e os restantes seis (6) selecionados para a tarefa, juntamente com os textos de compreensão oral e leitora da bateria MTL-BR. As narrativas orais foram gravadas em formato .mp3, em alta definição, com voz feminina, por microfone e com software próprio para gravação, com prosódia normal da língua, português padrão do Rio Grande do Sul. As narrativas escritas foram disponibilizadas impressas em papel sulfite A4 branco, com letra de forma em fonte Calibri, tamanho 16, na cor preta, parágrafo justificado, sendo uma página para cada uma.

${ }_{3}$ Disponível em: <http://143.107.183.175:22680/>. 
Após a audição ou a leitura silenciosa de cada narrativa, o examinador fez seis (06) perguntas acerca da macroestrutura (perguntas 1 a 3) e da microestrutura (perguntas 4 a 6) da narrativa. Os participantes poderiam ouvir ou ler as narrativas quantas vezes fossem necessárias. As perguntas deveriam ser respondidas oralmente, nas duas modalidades (ver ANEXo I-B). Os textos escritos não eram disponibilizados durante as respostas requeridas aos participantes, da mesma forma que os textos orais não poderiam ser ouvidos novamente após cada pergunta.

\subsection{Procedimentos para coleta e análise dos dados}

Primeiramente, a tarefa de compreensão leitora foi aplicada. Os quatro textos foram dados em sequência, com pequenos intervalos de 15 a 60 segundos entre um e outro. Após cada texto, eram lidas as questões de compreensão leitora, que deveriam ser respondidas oralmente, uma a uma, pelo participante. A instrução da tarefa de compreensão leitora foi semelhante à instrução da tarefa de compreensão oral. Não houve distribuição randômica da ordem dos textos, nem alterações na ordem de aplicação dessas tarefas.

Para a aplicação da tarefa de compreensão de narrativas orais, uma amostra de 10 segundos do texto de treino foi executada, com vistas à verificação do volume do áudio. Os participantes ouviram os áudios em aparelho celular smartphone ou tablet. Tais equipamentos foram utilizados para a audição devido à qualidade do volume, ausência de ruídos e facilidade de transporte. A aplicação das tarefas foi gravada em áudio por computador notebook posicionado sobre a mesa, a aproximadamente $50 \mathrm{~cm}$ do participante, de modo que não interferisse no contato visual com o aplicador. Como procedimento para a análise da tarefa de reconto, correspondente à pergunta de compreensão $\mathrm{n}^{\mathrm{o}}$ 1, dividimos as informações em informações principais (IP) e complementares (IC). 0 critério para estabelecer quais eram informações principais e quais eram complementares foi obtido a partir da avaliação dos juízes. Para a análise das respostas para as perguntas no 2 e noㅜ 3 , estabelecemos respostas corretas possíveis, também baseadas nas respostas dos juízes, contabilizando 1 ponto para cada informação correta lembrada, tal qual foi feito para o reconto. A soma do total de pontos nas questões de macroestrutura (1, 2 e 3 ) configurou a variável TOTALMACRO, utilizando CL para compreensão leitora e CO para compreensão oral, respectivamente. A pontuação total para a variável TOTALMACRO-CL era de 23 a 29 pontos e TOTALMACRO-CO de 24 a 25 pontos.

As respostas para as perguntas sobre microestrutura no 4, no 5 e no 6 foram contabilizadas em respostas corretas ou incorretas, considerando 1 ponto para cada uma lembrada corretamente. Cada texto possuía 3 perguntas sobre o nível microestrutural, totalizando (TOTALCLMICRO e TOTALCOMICRO) no máximo 9 pontos. A variável derivada da soma de pontos nas questões 4, 5 e 6 foi a TOTALMICRO-CL para compreensão leitora e TOTALMICRO-CO para compreensão oral.

O escore de compreensão total (TOTALCL para compreensão leitora e TOTALCO para compreensão oral) dos textos em cada modalidade foi dado mediante a soma dos índices totais de micro e macroestrutura. Nos três textos para a tarefa de compreensão leitora, o escore máximo foi de 86 pontos, já para os textos da tarefa de compreensão oral o escore máximo foi de 85 pontos.

$\mathrm{Na}$ análise, comparamos os índices obtidos nas variáveis TOTALMACRO (CL ao CO), TOTALMICRO (CL ao CO) e TOTALCL ao TOTALCO entre o grupo controle (GC) e grupo de participantes com LHE (GLHE). As variáveis foram descritas por médias, desvios padrão (dp) e percentuais. Foram considerados significativos os valores de $p$ menores do que 0,05 . A seguir, os resultados são apresentados com a devida discussão à luz da literatura revisada. 


\section{Resultados e discussão}

Em uma observação inicial, é possível afirmar que os grupos conseguiram realizar as tarefas, tanto de compreensão oral quanto escrita. Parece ter havido entendimento das instruções, visto que nenhum participante deixou de realizar as tarefas. Os textos de treino foram satisfatórios para a realização das atividades requeridas.

Na Tabela 3, observamos que a média nas questões de macroestrutura da compreensão leitora (TOTALMACRO-CL) foi maior no GC, mas essa diferença não é estatisticamente significativa. A quantidade de acertos dos grupos diferiu em aproximadamente 13,8 pontos, sendo a performance do GC superior ao GLHE. Ressaltamos que a pontuação máxima para TOTALMACRO-CL foi de 77 pontos. Considerando esse total, observamos que o GC obteve um escore de $48,7 \%$ enquanto o GLHE obteve $30,8 \%$. Uma diferença de $17,8 \%$ foi vista entre os grupos.

Tabela 3 - Resultados da comparação dos grupos GC e GLHE na compreensão leitora de macroestrutura (TOTALMACRO-CL)

\begin{tabular}{ccc}
\hline & Média $( \pm \mathbf{d p})$ & $\mathbf{P}$ \\
GC & $37,5( \pm 14,0)$ & 0,1 \\
GLHE & $23,7( \pm 8,4)$ & \\
\hline
\end{tabular}

GC: Grupo Controle; GLHE: Grupo com Lesão de Hemisfério Esquerdo; dp: Desvio padrão. Fonte: Autoras (2017).

Conforme os resultados, podemos afirmar que os grupos obtiveram o mesmo desempenho. Essa tendência pode estar em consonância com a literatura, a qual indica que os indivíduos com lesão utilizam os mesmos processos que os indivíduos sem lesão para a compreensão leitora de um texto.

Já na Tabela 4, são apresentados os dados de macroestrutura da compreensão oral (TOTALMACRO-CO). Conforme os dados fornecidos, observamos que as médias dos grupos são diferentes, tal qual ocorreu com as questões de macroestrutura de compreensão leitora. O GC apresentou um índice maior de acertos do que o GLHE, embora a diferença não seja observada estatisticamente. É possível notar uma tendência para que essa diferença seja significativa caso o número de participantes aumente $(p=0,07)$. A quantidade de pontos que distingue o GC do GLHE é de 11,2, sendo o GC com melhor desempenho. A pontuação máxima a ser obtida na tarefa era de 76 pontos. O GC obteve um escore de 53,9\% e o GLHE de 39,1\%. A diferença entre as performances entre eles foi de $14,8 \%$.

Tabela 4 - Resultados da comparação dos grupos na compreensão oral de macroestrutura

\begin{tabular}{ccc}
\hline & Média $( \pm \mathbf{d p})$ & $\mathbf{p}$ \\
GC & $41,0( \pm 5,0)$ & 0,076 \\
GLHE & $29,7( \pm 9,1)$ & \\
\hline
\end{tabular}

GC: Grupo Controle; GLHE: Grupo com Lesão de Hemisfério Esquerdo; dp: Desvio padrão. Fonte: Autoras (2017).

A princípio, não encontramos diferenças estatísticas significativas entre os grupos nas tarefas de macroestrutura nas duas modalidades investigadas. No entanto, apresentamos os resultados das modalidades oral e leitora em percentual na Tabela 5, a fim de pontuarmos algumas diferenças.

Tabela 5 - Comparação da compreensão leitora e oral de macroestrutura em percentual

\begin{tabular}{ccccc}
\hline Grupo & $\begin{array}{c}\text { TOTAL } \\
\text { MACRO-CL }\end{array}$ & Diferença \% & $\begin{array}{c}\text { TOTAL } \\
\text { MACRO-CO }\end{array}$ & Diferença \% \\
GC & $48,7 \%$ & $17,86 \%$ & $53,94 \%$ & $14,8 \%$ \\
GLHE & $30,84 \%$ & & $39,14 \%$ & \\
\hline
\end{tabular}

GC: Grupo Controle; GLHE: Grupo com Lesão de Hemisfério Esquerdo; TOTALMACRO-CL: Macroestrutura da compreensão leitora; TOTALMACRO-CO: Macroestrutura da compreensão oral. Fonte: Autoras (2017). 
Ao comparar a quantidade de acertos nas tarefas de macroestrutura, observamos que o GC obteve uma média de $51,32 \%$ de acertos nas variáveis TOTAL MACRO-CL e TOTAL MACRO-CO. Já o GLHE obteve uma média de $34,99 \%$. Considerando que os grupos não diferiram estatisticamente nas médias de acertos das tarefas de macroestrutura oral e leitora, observamos que a macroestrutura do GLHE pode estar preservada, tal qual posto por Dressler e Pleh (1988), Glosser e Deser (1991), Huber (1990), Ulatowska et al. (1983), Ulatowska e Chapman (1991), e Ulatowska, North e Macaluso-Haynes (1981).

Entretanto, na modalidade oral, vimos que ambos os grupos obtiveram melhor desempenho nas questões de macroestrutura oral, sugerindo que os indivíduos podem se beneficiar mais da modalidade oral que da modalidade escrita para a compreensão da macroestrutura. Pesquisas sugerem que os indivíduos com LHE poderão ser beneficiados na modalidade oral pela frequência superior com que ela é usada em relação à compreensão leitora de texto (ORTIZ, 2010). Além disso, nas populações com baixa escolaridade, a modalidade escrita pode apresentar-se como um obstáculo (ORTIZ; OSBORN; CHIARI, 1993). Considerando a amostra deste estudo, que se trata, na maioria, de indivíduos com baixa escolaridade, pode-se afirmar que a modalidade escrita foi mais desafiadora para os participantes. A partir disso, tomaremos como critério um nível de escolaridade mais alto, bem como a utilização de um instrumento de leitura de palavras e pseudopalavras, para caracterizar a leitura dos participantes.

Ainda, qualitativamente, ao realizar as tarefas que requeriam a construção da macroestrutura, os GLHE pareceram mais concisos que os controles em relação à quantidade de palavras, frases, encadeamento de ideias, utilização de conectivos e utilização de nomes próprios. No Quadro 1, duas respostas à pergunta no 1 de um participante controle e um com lesão encontram-se transcritas.
Quadro 1 - Exemplos transcritos de narrativas orais

\begin{tabular}{|l|l|}
\hline Participante GC & Participante GLHE \\
... ah:: é que a Rosana tava tomando banho... & a::.... ela foi pr/tomar banho e.: queimou a \\
o chuveiro fu/queimou... ela sentiu cheiro de & resistência do chuveiro... aí ela.... se secou e foi \\
queimado ((tosse)) ... ai a resistência tinha & numa azência que ele/ ferragem assim... que ele \\
queimado... aí ela trocou de roupa e foi na & comprou uma resistência e tinha em casa mais \\
ferragem comprar outra resistência... ai como & umas ferramentas que precisava... e trocou a \\
ela não entendia... pediu pro rapaz a informação & resistência... e.: aí ela conseguiu tomar banho... \\
neh:: para como teria que trocar o chuveiro & \\
trocar a resistência... aí chegou em casa pegou & \\
as ferramentas e trocou a resistência e voltou a & \\
tomar banho quente &
\end{tabular}

Fonte: Autoras (2017).

Observamos que foi recorrente nas respostas dos participantes do grupo GLHE a ausência de nomes próprios, presentes em todas as narrativas. Como referentes para esses substantivos, os participantes utilizaram os termos "ele", "ela", "a mulher", "o cara" ou até mesmo seus próprios nomes, quando lhes parecia uma situação em que se encaixavam. Observamos ainda que o participante do GLHE omitiu no seu reconto o pedido de ajuda para o atendente da ferragem, presente na transcrição do participante GC. Além disso, o participante do GLHE não menciona quem seria o referente para "ele", podendo ser uma referência ao atendente da ferragem ou à personagem principal da narrativa. Ainda, há a menção da palavra "agência" (azência), seguida de posterior correção e troca pela palavra ferragem. Os participantes do GLHE demonstraram maior disposição quando o assunto lhes era familiar, incluindo histórias pessoais nas respostas às perguntas. Por fim, destacamos o encadeamento de ideias da narrativa, com início, meio e fim apresentadas pelos participantes GLHE.

Diferenças estatísticas entre a compreensão oral e leitora de macroestrutura não foram encontradas. Todavia, fatores como quantidade de palavras, quantidade de repetições da mesma palavra e quantidade de 
frases são vistos como diferenças qualitativas entre os grupos, o que pode, futuramente, apresentar-se relevante estatisticamente.

A partir da Tabela 6, observamos que as médias de acertos nas questões de compreensão leitora de microestrutura (TOTALMICRO-CL) são distintas para os grupos GC e GLHE, mas não foram significativas estatisticamente. A quantidade de acertos dos grupos diferiu pouco, com 1,3 pontos de diferença. O GC teve melhor desempenho que o GLHE. Ressaltamos que a pontuação máxima possível para as tarefas de compreensão leitora de microestrutura era de 9 pontos. Considerando esse número, observamos que tanto o GC quanto o GLHE obtiveram aproveitamento de mais de $50 \%$ nesse quesito. O GC teve $94,4 \%$ de acertos, ao passo que o GLHE obteve um escore de $80 \%$. 0 índice de diferença entre os grupos de $14,4 \%$ não foi significativo estatisticamente.

Tabela 6 - Resultados da comparação entre os grupos GC e GLHE na compreensão leitora de microestrutura (TOTALMICRO-CL)

\begin{tabular}{ccc}
\hline & Média $( \pm d \mathbf{d p})$ & $\mathbf{p}$ \\
GC & $8,5( \pm 1,0)$ & 0,1 \\
\hline
\end{tabular}

GC: Grupo Controle; GLHE: Grupo com Lesão de Hemisfério Esquerdo; dp: Desvio padrão. Fonte: Autoras (2017).

Os dados sobre a compreensão oral da microestrutura também mostram um índice de acertos de mais de $50 \%$ para ambos os grupos. O GC apresentou mais elementos da narrativa que o GLHE nas duas modalidades, mas não houve diferenças significativas. O GC obteve um escore de 91,1\% e o GLHE de 83,3\%. A diferença entre eles totalizou 7,8\%, não configurando diferença estatística. Observamos uma sutil diminuição no desempenho do GC quando comparamos as modalidades (TOTALMICRO-CL $=8,5$ e
TOTALMICRO-CO = 8,2), com uma variação de 0,3 pontos. Também ocorreu um sutil aumento no desempenho do GLHE na modalidade oral (GLHE $\mathrm{CL}=7,2$ e GLHE $\mathrm{CO}=7,5$ ), com a mesma variação de 0,3 pontos. Essa variação de 0,3 pontos não foi estatisticamente significativa, porém pode indicar uma tendência para uma melhor performance na modalidade oral da microestrutura, tal qual ocorre na modalidade oral da macroestrutura, conforme mostra a Tabela 7.

Tabela 7 - Resultados da comparação entre os grupos GC e GLHE na compreensão oral de microestrutura (TOTALMICRO-CO)

\begin{tabular}{ccc}
\hline & Média $( \pm \mathbf{d p})$ & $\mathbf{p}$ \\
GC & $8,2( \pm 0,5)$ & 0,2 \\
\hline
\end{tabular}

GC: Grupo Controle; GLHE: Grupo com Lesão de Hemisfério Esquerdo; DP: Desvio padrão. Fonte: Autoras (2017)

A literatura sobre compreensão de micro e macroestrutura de narrativas aponta que a compreensão de macroestrutura costuma ser melhor do que a de microestrutura. Tanto as populações de indivíduos saudáveis quanto as de indivíduos com lesão parecem utilizar os mesmos processos de compreensão, construindo uma ideia geral das narrativas e omitindo detalhes. Para Brookshire e Nicholas (1984), detalhes distintos têm efeitos diferentes na compreensão. Eles salientam que fatores como números, horas, locais com nomes próprios, meses, estações do ano, partes do dia (especialmente o termo "noite") e dias da semana são os que mais causam problemas de compreensão. Esses foram requeridos nas tarefas de microestrutura deste estudo e não apresentaram desafios aos participantes.

Considerando a literatura consultada para a realização deste estudo, observamos tantas diferenças em suas metodologias que dificultam a 
comparação de resultados. Entretanto, os dados obtidos nesta pesquisa podem fornecer mais informações sobre o desempenho de participantes com diferentes locais de lesão por acidente vascular isquêmico em tarefas de compreensão de macro- e microestrutura.

A compreensão total de cada modalidade deu-se mediante a soma da pontuação das questões de micro e macroestrutura (TOTALMACRO e TOTALMICRO), conforme mostra a Tabela 8.

Tabela 8 - Índices de compreensão total na modalidade leitora (TOTALCL) e oral (TOTALCO)

\begin{tabular}{|c|c|c|c|c|}
\hline & $\begin{array}{c}\text { TOTALCL } \\
\text { (Média/dp) }\end{array}$ & $\mathbf{P}$ & $\begin{array}{c}\text { TOTALCO } \\
\text { (Média/dp) }\end{array}$ & $\mathbf{p}$ \\
GC & $46,2(14,6)$ & 0,1 & $49,2(4,9)$ & 0,07 \\
GLHE & $31(8,6)$ & $37,2(9,9)$ & \\
\hline
\end{tabular}

GC: Grupo Controle; GLHE: Grupo com Lesão de Hemisfério Esquerdo; dp: Desvio padrão. Fonte: Autoras (2017)

Notamos uma sutil diferença na modalidade oral, tanto na micro quanto na macroestrutura. A literatura sobre o assunto é bastante dividida. As pesquisas que apontam diferenças em relação a essas modalidades explicam que tais diferenças se devem a alguns fatores como frequência dos hábitos socioculturais.

Um dos hábitos socioculturais que pode influenciar na compreensão de textos é a leitura. Ulatowska et al. (2013) explicam que os indivíduos com LHE apresentam menos dificuldades em tarefas de reconto quando possuem hábitos de leitura frequentes, influenciados pelo alto grau de instrução e profissões que requerem muita leitura. Então, pode-se dizer que tanto os anos de instrução, quanto os hábitos de leitura, bem como a exigência dessas habilidades devido ao tipo de profissão, têm papel relevante no desempenho da compreensão em indivíduos com lesões. Essas variáveis podem funcionar como uma estratégia compensatória para os prejuízos decorrentes das lesões cerebrais. Inclusive, González-Fernández et al. (2011) afirmam que pessoas com mais de 12 anos de instrução tendem a ler mais frequentemente e a se envolverem mais em atividades com maior engajamento cognitivo. 0 acesso frequente às palavras escritas pode fortalecer sinapses associadas com o processo de compreensão e pode aumentar o número de sinapses capazes de lidar com esse processo, tornando os indivíduos menos vulneráveis aos prejuízos linguísticos advindos de lesões cerebrais.

Os participantes desta pesquisa trataram-se de indivíduos, no geral, com baixa escolaridade e com profissões que não requeriam hábitos de leitura frequentes. Destacamos que as profissões eram: costureira, mecânico, vendedora, motorista, do lar e auxiliar de serviços gerais. Refletindo acerca do que a literatura destaca sobre a influência de hábitos socioculturais como a leitura e a profissão na compreensão de narrativas, observamos que este fato pode ter tido efeito sob os resultados deste estudo. Ainda que dois (02) participantes do grupo GC tenham apresentado Ensino Médio (escolaridade superior ao restante da amostra), acreditamos que, devido também ao número reduzido de participantes, não foram encontradas diferenças expressivas no desempenho das tarefas de compreensão.

Considerando que este estudo foi um piloto com um número limitado de participantes, ainda é prematuro afirmar que há diferenças na compreensão dos níveis micro e macroestruturais de narrativas entre os grupos, especialmente no que tange às modalidades. É essencial um número mais expressivo de participantes, o controle de mais variáveis como a atenção, mais testes linguísticos e a distribuição randômica das tarefas. Tais quesitos serão observados nas pesquisas futuras deste grupo de pesquisa. 


\section{Considerações finais}

O objetivo da pesquisa foi investigar a compreensão oral e leitora dos níveis micro e macroestruturais de narrativas, por meio de um estudo piloto incluindo indivíduos com LHE e indivíduos saudáveis. Conforme a literatura, haveria uma tendência dos participantes com LHE de compreenderem as narrativas utilizando os mesmos processos que os controles. Esses processos estariam baseados na melhor compreensão da macroestrutura.

Os resultados preliminares obtidos neste estudo mostraram que o grupo com LHE apresentou desempenho similar ao GC em todas as tarefas. Ao comparar o desempenho nas questões de macroestrutura, observamos diferenças qualitativas nas respostas, como na quantidade de palavras, frases e uso de nomes próprios. Houve uma sutil diferença ao comparar a macroestrutura oral entre os grupos. Tanto o GC quanto o GLHE obtiveram melhor desempenho nas tarefas orais.

Em relação ao nível microestrutural da compreensão leitora e oral, os grupos não se diferenciaram. Tanto o GC quanto o GLHE apresentaram desempenho acima de $80 \%$, não diferindo estatisticamente entre as modalidades. O GC apresentou uma sutil diminuição na performance da compreensão leitora para a oral, enquanto o GLHE apresentou um pequeno aumento na compreensão oral. Para explicar essa ocorrência, ainda que sutil, trouxemos dados da literatura sobre a frequência de hábitos socioculturais.

Por fim, destacamos que, como se tratou de um estudo piloto, quesitos tais como um número maior de participantes deva ser observado em pesquisas futuras. Por enquanto, podemos afirmar que este estudo piloto auxiliou no refinamento da metodologia da pesquisa e foi uma oportunidade de reflexão acerca dos procedimentos realizados na coleta e análise dos dados. Além disso, foi uma oportunidade para testar, avaliar, revisar e aprimorar os textos e perguntas desenvolvidas para o projeto, bem como, o momento certo para averiguar possíveis pontos fracos e problemas em potencial.

Dentre as limitações que encontramos estão: 1) a necessidade de randomização dos textos e dos questionários; 2) a necessidade de controle dos hábitos socioculturais e profissão dos participantes; 3) a necessidade de um controle maior da escolaridade, com teste de leitura de palavras e pseudopalavras, por exemplo; 4) a necessidade de aprimoramento da análise estatística; 5) a necessidade de diminuir a quantidade de textos a serem lidos/ouvidos, devido à longa duração da coleta; 6) a necessidade de estabelecer um local de coleta adequado e que propicie a gravação de áudio com menos ruídos e 7) a necessidade de aprimoramento da instrução dada aos participantes. Embora algumas limitações tenham sido enumeradas, acreditamos que a amostra tenha oferecido dados interessantes sobre essa população clínica específica, instigando a continuidade desta pesquisa.

Acreditamos que a presente pesquisa e futuros trabalhos nessa temática possam contribuir para o aumento de dados sobre a compreensão de narrativas por indivíduos com LHE, auxiliando em um prognóstico mais específico da compreensão da linguagem para essa população. Também esperamos ressaltar a necessidade de inserção de narrativas na confecção de baterias para a avaliação da linguagem e, por fim, contribuir para o âmbito interdisciplinar entre a linguística, a neurociência e a fonoaudiologia, permitindo a discussão sobre a compreensão de narrativas por indivíduos com LHE com o auxílio da perspectiva dessas áreas.

\section{Referências}

ALMEIDA, Osvaldo P.; ALMEIDA, Shirley A. Short versions of the geriatric depression scale: a study of their validity for the diagnosis of a major depressive episode according to ICD-10 and DSM-IV. International Journal of Geriatric Psychiatry, v. 14, n. 10, p. $858-865,1999$. 
ALUISIO, Sandra et al. Readability assessment for text simplification. Proceedings of the NAACL HLT 2010 Fifth Workshop on Innovative Use of NLP for Building Educational Applications - Association for Computational Linguistics, 2010. p. 1-9.

ASSOCIAÇÃO BRASILEIRA DE EMPRESAS DE PESQUISA. Critério de classificação econômica Brasil, 2015. Disponível em: <http://www.abep.org/criterio-brasil>. Acesso em: 20 set. 2016.

BRITTO, Gilberto N. O. et al. Lateral preferences in Brazilian adults: an analysis with the Edinburgh Inventory. Cortex, v. 25, n. 1, p. 403-415, 1989.

BROOKSHIRE, Robert H.; NICHOLAS, Linda. E. Comprehension of directly and indirectly stated main ideas and details in discourse by brain-damaged and non-brain-damaged listeners. Brain and language, v. 21, n. 1, p. 21-36, 1984.

CHAPMAN, Sandra Bond; ULATOWSKA, Hanna K. Methodology for discourse management in the treatment of aphasia. Clinics in Communication disorders, v. 2, n. 1, p. 64-81, 1992.

CHAVES, Márcia Lorena Fagundes; IZQUIERDO, Ivan. Differential diagnosis between dementia and depression: a study of efficiency increment. Acta Neurologica Scandinavica, v. 85, n. 6, p. 378-382, 1992

DIXON, Roger A. et al. Text recall in adulthood as a function of level of information, input modality, and delay interval. Journal of Gerontology, n. 37, p. 358-364, 1982.

DRESSLER, Wolfgang U.; PLEH, Csaba. On text disturbances in aphasia. In: DRESSLER, W.U., STARK, J. A. (Ed.). Linguistic analyses of aphasic language. New York: SpringerVerlag, 1988. p. 151-178.

FONSECA, Rochele Paz et al. Métodos em avaliação neuropsicológica. In: LANDEIRAFERNANDEZ, Jesus; FUKUJIMA, Sergio Sheiji (Org.). Métodos em neurociência. São Paulo: Manole, 2012. p. 266-296

GERGEN, Kenneth J.; GERGEN, Mary M. Narrative and the Self as Relationship. Advances in Experimental Social Psychology, v. 21, n. C, p. 17-56, 1988.

GLOSSER, Guila; DESER, Toni. Patterns of discourse production among neurological patients with fluent language disorders. Brain and Language, v. 40, n. 1, p. 67-88, 1991.

GONZALEZ-FERNANDEZ, Marlís et al. Formal education, socioeconomic status, and the severity of aphasia after stroke. Archives of Physical Medicine and Rehabilitation, v. 92, n. 11, p. 1809-1813, 2011.

GREEN, Ruth. Remembering ideas from text: the effect of modality of presentation. British Journal of Educational Psychology, n. 51, p. 83-89, 1981.
HABERMAS, Tilmann; BLUCK, Susan. Getting a life: The emergence of the life story in adolescence. Psychological Bulletin, v. 126, p. 748-769, 2000.

HUBER, Walter. Text comprehension and production in aphasia: Analysis in terms of micro- and macrostructure. In: JOANETTE, Yves; BROWNELL, Hiram H. (Ed.). Discourse ability and brain damage: Theoretical and empirical perspective. New York, NY: SpringerVerlag, 1990. p. 154-179.

KAMAKURA, Wagner; MAZZON, José Afonso. Estratificação Socioeconômica e Consumo no Brasil. São Paulo: Edgard Blucher, 2013.

KINTSCH, Walter; KOZMINSKY, Ely. Summarizing stories after reading and listening. Journal of Educational Psychology, v. 69, n. 5, p. 491-499, 1977.

KOCH, Ingedore Villaça; CUNHA-LIMA, Maria Luiza. Do cognitivismo ao sociocognitivismo. In: MUSSALIN, Fernanda; BENTES, Anna Christina. Introdução à linguística: fundamentos epistemológicos, v. 3. São Paulo: Cortez, 2004. p. 251-300.

LUND, Randall J. A comparison of second language listening and reading comprehension. The Modern Language Journal, n. 75, p. 196-204, 1991.

MOYER, Jessica Emily. "Teens today don't read books anymore": A study of differences in comprehension and interest across formats. 2011. 184f. Tese (Doutorado em Filosofia) - Universidade de Minnesota, MN, Minnesota, 2011.

NICHOLAS, Linda E.; BROOKSHIRE, Robert H. A system for quantifying the informativeness and efficiency of the connected speech of adults with aphasia. Journal of speech and hearing research, v. 36, n. 2, p. 338-350, 1993.

ORTIZ, Karin Zazo. Distúrbios Neurológicos Adquiridos: Linguagem e Cognição, 2. ed, Baurueri, SP: Manole, 2010.

ORTIZ, Karin Zazo; OSBORN, Ellen; CHIARI, Brasília Maria. O teste M1-alpha como instrumento para avaliação da afasia. Pró-Fono; v. 5, n. 1, p. 23-9, 1993.

PARENTE, Maria Alice de Mattos Pimenta et al. Bateria Montreal-Toulouse de Avaliação da Linguagem - Bateria MTL-Brasil. São Paulo: Vetor, 2016.

PAWLOWSKI, Josiane et al. The influence of reading and writing habits associated with education on the neuropsychological performance of Brazilian adults. Reading \& Writing, n. 25, p. 2275-2289, 2012.

PIERCE, Robert. S.; GROGAN, Susan. Improving listening comprehension for narratives. Clinics in communication disorders: Aphasia. Reading, v. 2, n. 1, p. 54-63, 1992. 
ROGOWSKY, Beth A.; CALHOUN, Barbara M.; TALLAL, Paula. Does Modality Matter? The Effects of Reading, Listening and Dual Modality on Comprehension. SAGE Open, v. 6, n. 3, 2016.

SACHS, Jacqueline S. Memory in reading and listening to discourse. Memory \& Cognition, n. 2, p. 95-100, 1974.

SPRINGER, Luise. Therapeutic approaches in aphasia rehabilitation. In: STEEMER, Brigitte; WHITAKER, Harry. Handbook of the neuroscience of language. USA: Academic Press, 2008. p. 397-405.

ULATOWSKA, Hanna K.; CHAPMAN, Sandra Bond. Macrostructure in aphasia. In: BLOOM, Ronald L. et al. (Ed.). Discourse analysis and applications: Studies in adult clinical populations. Hillsdale, NJ: Lawrence Erlbaum, 1994. p. 29-46.

ULATOWSKA, Hanna K.; CHAPMAN, Sandra Bond. Language and studies and dementia. In: LUBINSKI, R. (Ed.). Dementia in communication: research and clinical implications. Philadelphia: BC Decker, 1991. p. 90-112.

ULATOWSKA, Hanna K. et al. Production and comprehension in aphasia: gains and pitfalls in using macrostructure tasks in Aesop's fables. International Journal of Speech-Language Pathology, v. 15, n. 6, p. 634-642, 2013.

Production of narrative discourse in aphasia. Brain and language, v. 19, n. 2 p. 317-334, 1983.

ULATOWSKA, Hanna K.; NORTH, Alvin J.; MACALUSO-HAYNES, Sara. Production of narratives and procedural discourse in aphasia. Brain and Language, v. 13, p. 345-371, 1981.

ULATOWSKA, Hanna K.; SADOWSKA, Maria. Some observations on aphasic texts. In: HWANG, S. J., MERRIFIELD, W. R. (Ed.). Language in context: Essays for Robert E. Longacre. Arlington: The Summer institute of Linguistics and the University of Texas at Arlington, 1992. p. 51-66.

van DEN BROEK, Paul. Comprehension and memory of narrative texts: Inferences and coherence. In: GERNSBACHER, Morton Ann (Org.). Handbook of psycholinguistics, San Diego, CA: Academic Press, 1994. p. 539-588.

van DIJK, Teun A. Narrative macrostructures: cognitive and logical foundations. PTL: A Journal for Descriptive Poetics and Theory of Literature, v. 1, p. 547-568, 1976.

WEGNER, Martha L.; BROOKSHIRE, Robert H.; NICHOLAS, L. E. Comprehension of main ideas and details in coherent and noncoherent discourse by aphasic and nonaphasic listeners. Brain and Language, v. 21, n. 1, p. 37-51, 1984.

\section{Anexo}

\section{ANEXO I}

Exemplo de estória e perguntas da tarefa linguística

(A) Marcela e a gripe

Ontem à noite, Marcela tomou um banho de chuva na volta da escola e começou a apresentar sintomas de gripe. Após ter tomado um chá em vão, Marcela liga para sua mãe Luísa, que rapidamente providencia uma sopa deliciosa e revigorante. No entanto, ao ver que os sintomas da gripe não passam, Luísa decide levá-la ao médico. Quando chegam ao consultório médico, elas esperam quatro horas para serem atendidas. Luísa pensa em ir embora mas precisa que a filha seja medicada. Após tanta espera, Marcela é atendida com dedicação pelo Dr. Rafael, que lhe recomendou repouso. Dr. Rafael também pediu desculpas pela demora no atendimento, pois tinha estado acamado com gripe.

(B) 1. O que aconteceu nessa estória?

2. Por que Luísa levou Marcela ao médico?

3. Por que Marcela estava com gripe?

4. Luísa é mãe de Marcela?

5. Luísa esperou pouco tempo para ser atendida?

6. Dr. Rafael demorou porque estava doente? 